\title{
Clarifying Core Content of Goals of Care Discussions
}

\author{
Lauris C. Kaldjian, $M D, P h D$ D
}

Department of Internal Medicine, and Program in Bioethics and Humanities, University of lowa Carver College of Medicine, lowa City, IA, USA.

KEY WORDS: goals of care; patient-physician communication; medical education.

J Gen Intern Med 35(3):913-5

DOI: $10.1007 / \mathrm{s} 11606-019-05522-5$

(c) Society of General Internal Medicine 2019

\begin{abstract}
A recent review ${ }^{1}$ reveals what many of us observe in practice: clinicians use the phrase "goals of care" in a variety of ways, often with a definition more assumed than revealed. The phrase is of particular importance when it is used to refer to a certain kind of conversation between a clinician and patient (or family), often called a "goals of care discussion." Sometimes the need for such a discussion is a clinician's shorthand way of indicating it is time to clarify a patient's code status or shift the focus of care to palliation. At other times, goals of care discussions refer to the need for a specific approach to conversation about a more general plan of care. For instance, one task force recommends that goals of care discussions in serious illness should address not only prognosis and goals, but also decision-making preferences, family involvement, fears, function, and tradeoffs. ${ }^{2}$ Others suggest that goals of care discussions should help patients' establish "agreed on, desired health expectations that are appropriate, documented and communicated," ${ }^{3}$ that these conversations should clarify patients' values, goals, and priorities in light of information about their diagnosis, prognosis, and treatment options, ${ }^{4}$ or that these conversations should include a leading emphasis on the decision that needs to be made and the likelihoods of different possible outcomes regarding sur-
\end{abstract} vival, symptoms, and functional ability. ${ }^{5}$

Such differences in approach are reflected in another recent review that assessed elements of goals of care discussions and their operationalization ${ }^{6}$ and concluded that consensus is lacking on the question of content. Moreover, some authors suggest these discussions may be less about goals directed at treatment decisions, and more about emotional issues, management of symptoms, and rapport-building. ${ }^{4}$ In defense of such diversity, we can accept the need for flexibility in the content of goals of care discussions given the many differences among patients, their conditions, and their circumstances. But we also do well to consider the content that should

Received September 20, 2019

Accepted October 25, 2019

Published online November 11, 2019 form the consistent core of these conversations when significant clinical decisions need to be made - especially when we are trying to explain and model for trainees practices that are clinically meaningful and feasible within the demands of clinical practice.

To this end, based on literature and experience, I suggest the core content of goals of care discussions should include diagnosis, prognosis, goals of care, treatment or test, burdens of treatment or test, and probabilities of outcomes with or without treatment or test (Fig. 1). These are key dimensions of decision making we need to comprehend and remember to communicate so that shared decision making with patients is facilitated by shared understanding. Not surprisingly, these dimensions have much in common with elements of informed consent. By making the patient's most important goals of care the conversational focal point, decision making is patient-centered, purpose-oriented, and premised on the patient's beliefs, values, priorities, preferences, and circumstances. Specific goals of care can be elicited by open-ended and closedended questions regarding goals, expectations, or hopes. For many patients, preferred goals of care will fall within one of the following categories: cure, live longer, improve or maintain function, comfort, achieve life goals, or provide support for family. ${ }^{7}$ The core content I suggest endeavors to capture what is most essential and feasible in other approaches, and to keep goals at the center of goals of care discussions. This content is fundamental enough to be consistent with the risks, benefits, and likelihoods of informed consent discussions. As a clinician and teacher, I believe this approach satisfies the need for a clinically practical way to articulate (and sometimes even diagram in front of patients) the dimensions that guide reasoning about decisions, and to communicate that these different dimensions are inter-related and therefore need to be taken together.

One of the hallmarks of a successful goals of care discussion is that it allows everyone to take a step back from the intense focus on a specific intervention (such as cardiopulmonary resuscitation ${ }^{8}$ ) by considering the broader purpose and context of that intervention. While interventionoriented decision making tends to focus on the most readily available means of addressing the most immediate medical problem, goal-oriented discussions take a larger, longer view. Framed by goals of care, conversation about decision making moves the question from "Would you like us to do this, or not?" to "Will this help us achieve your goals of care, or not?" In this way, goal-oriented decision making draws our attention 
Burdens of treatment or test

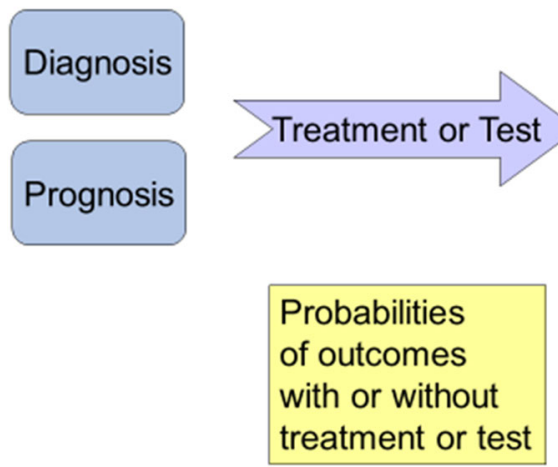

Figure 1 Core content of a goals of care discussion.

to the priority of ends over means, and it reminds us why every service in the hospital (not just palliative care) should be a "goals of care" service.

The problem with intervention-focused discussions was brought home to me one day when I was attending on an inpatient medical service. We were caring for an incapacitated man whose complicated and tenuous medical condition (which included $C$. difficile colitis and toxic megacolon) had already led his adult son to authorize a do-not-resuscitate order. On the third day of hospitalization our patient developed tachypnea. Diagnostic testing suggested the possibility of a pulmonary embolism. We were wondering whether we should rule out a deep vein thrombosis (DVT) with a view toward the possibility of placing an inferior vena cava (IVC) filter, if needed. We approached the patient's bedside to speak with his son. Our very capable, well-intentioned, but interventionfocused resident explained our clinical concerns about a blood clot in the patient's lung and then asked the patient's son two questions: "Do you want your father to have an IVC filter if we find a DVT, and do you want him to be transferred to the ICU if he deteriorates?"

At that moment I realized we were failing to make these intervention-focused questions sufficiently navigable for the son because we were dislocating these questions from a context that would make them meaningful - a context guided by goals, prognosis, probabilities of outcomes, and burdens of treatment. It was an important teaching moment and an opportunity to improve the process of care, so I gently "paused" the conversation and excused the team from the patient's room. After finding a place for the team to speak in private, we discussed the need to place such interventional questions within a goal-oriented framework. We then returned to the patient's room and continued the process of shared decision making with goals explicitly in view. This allowed the son to make an informed, purposeful decision that he believed was best for his father because it was the option most likely to achieve the goals of care that were most important.
The key dimensions of decision making that guide goals of care discussions are not only relevant in healthcare. While their application to medicine is particularly obvious, the roots of these dimensions are so fundamental to reasoning that their ramifications are perceived in any clear process of deliberation about any significant decision. We can illustrate this point with a medical student analogy. A medical student is a human being who wants and needs to do something in life (diagnosis) and anticipates a future in which to act and accomplish (prognosis). A medical student wants to help people be healthy (goal) and decides that medical school (treatment) is the best means to that worthwhile end. A medical student has had to seriously consider the direct and indirect demands of medical school (burdens) and the likelihood of being accepted to medical school and completing medical training (probabilities).

In teaching medical students, I have found this analogy helpful in driving home the nature and interrelationships of these key dimensions of decision making. In particular, I have found it useful for emphasizing that a treatment (like medical school) is never an end in itself, and that goals of care - as important as they are - must always be situated within a landscape formed by a patient's diagnosis and prognosis, the probabilities of outcomes related to different possible treatments, and the burdens that may accompany those treatments.

Seen from a historical perspective, the key dimensions of decision making that guide goals of care discussions can be understood as a part of the virtue of practical wisdom, ${ }^{9}$ a character trait that has been valued in human society since ancient times. Aristotle called it phronesis and Aquinas called it prudentia. When we and our patients have this and other virtues, we can engage in shared decision making in ways that are informed by biological and technical realities and guided by ethical values. And the central practical concern will be this: to identify and discuss goals that should be pursued and the means most likely to accomplish them. Such discussions should allow us to understand our patients well enough to 
know what matters most to them. Such discussions should also allow our patients to understand the information they require to make good decisions that harmonize with their values and needs. To accomplish this mutual understanding, we should be sure to cover a certain amount of conversational ground. The core content emphasized in this brief essay offers a way to describe the most prominent topography of that ground.

Corresponding Author: Lauris C. Kaldjian, $M D, P h D$; Department of Internal Medicine, and Program in Bioethics and HumanitiesUniversity of Iowa Carver College of Medicine, Iowa City. IA, USA (e-mail: lauris-kaldjian@uiowa.edu).

\section{REFERENCES}

1. Edmonds KP, Ajayi TA. Do we know what we mean? An examination of the use of the phrase "goals of care" in the literature [published online July 30, 2019]. J Palliat Med. doi:https://doi.org/10.1177/1049909119867046.
2. Bernacki RE, Block SD; American College of Physicians High Value Care Task Force. Communication about serious illness care goals: a review and synthesis of best practices. JAMA Intern Med. 2014;174(12): 1994-2003.

3. Stanek S. Goals of care: a concept clarification. J Adv Nurs. 2017;73(6): 1302-1314.

4. Schulman-Green D, Smith CB, Lin JJ, Feder S, Bickell NA. Oncologists and patients' perceptions of initial, intermediate, and final goals of care conversations. J Pain Symptom Manage. 2018;55(3): 890-896.

5. Fried TR. Communication about treatment options and shared decision making in the intensive care unit. JAMA Int Med. 2019;179(5):684-685.

6. Myers J, Cosby R, Gzik D et al. Provider tools for advance care planning and goals of care discussion: a systematic review. Am J Hosp Palliat Care. 2018;35(8):1123-1132.

7. Kaldjian LC, Curtis AE, Shinkunas LA, Cannon KT. Goals of care toward the end of life: a structured literature review. Am J Hosp Palliat Care. 2009;25(6):501-511.

8. Breu AC. Clinician-patient discussions of successful CPR - the vegetable clause. JAMA Intern Med. 2018;178(10):1299-1300.

9. Kaldjian LC. Practicing Medicine and Ethics: Integrating Wisdom, Conscience, and Goals of Care. New York: Cambridge University Press; 2014.

Publisher's Note Springer Nature remains neutral with regard to jurisdictional claims in published maps and institutional affiliations. 